\title{
AIAA 2004-2544
}

Three-dimensional instabilities of compressible flow over open cavities: direct solution of the BiGlobal eigenvalue problem.

V. Theofilis

Escuela Técnica Superior Ingenieros Aeronáuticos, Universidad Politcnica de Madrid,

Pza. Cardenal Cisneros 3, E-28040 Madrid, SPAIN

T. Colonius

Division of Engineering and Applied Science, California Institute of Technology, Pasadena CA 91125, USA

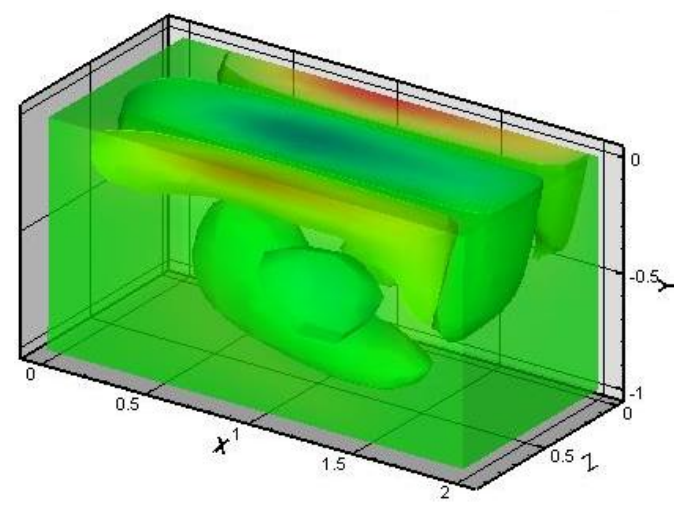

\section{4th Fluid Dynamics Conference and Exhibit June 28 - July 1, 2004 / Portland, Oregon}




\title{
Three-dimensional instabilities of compressible flow over open cavities: direct solution of the BiGlobal eigenvalue problem.
}

\author{
V. Theofilis \\ Escuela Técnica Superior Ingenieros Aeronáuticos, \\ Universidad Politcnica de Madrid, \\ Pza. Cardenal Cisneros 3, E-28040 Madrid, SPAIN \\ T. Colonius \\ Division of Engineering and Applied Science, \\ California Institute of Technology, \\ Pasadena CA 91125, USA
}

\begin{abstract}
We report progress in our ongoing effort to compute and understand the instabilities of open cavity flows from incompressible to supersonic speeds. We consider three-dimensional instabilities of nominally two dimensional (spanwise homogeneous) cavity flows (BiGlobal instabilities). Experiments, DNS/LES computations, and preliminary instability computations have shown that the modes of oscillation are influenced by complex interactions between the shear layer and the recirculating flow within the cavity. We present here a framework for computation of the two-dimensional eigenvalue problem for the compressible open cavity. We validate the numerical scheme by computing several canonical flows: square duct flow, boundary layers at speeds from incompressible to supersonic, and two-dimensional parallel shear layers. We present preliminary results for the three-dimensional modes of the compressible open cavity flow with length-to-depth ratio of two at a Mach number of 0.325 .
\end{abstract}

\section{Nomenclature}

Abbreviations

DNS direct numerical simulation

EVP eigenvalue problem

Latin symbols

c.c. complex conjugate

i imaginary unit

$L_{z} \quad$ periodicity length in the $z$-direction

$M \quad$ Mach number

$\overline{\mathbf{q}}, \tilde{\mathbf{q}} \quad$ basic, disturbance flow vector

$\hat{\mathbf{q}} \quad$ amplitude functions of linear disturbances

Re Reynolds number

$t \quad$ time

$(x, y, z) \quad$ cartesian coordinates

Calligraphic symbols

\begin{tabular}{ll}
\hline $\mathcal{D}_{x}, \mathcal{D}_{y}$ & $\partial / \partial x, \partial / \partial y$ \\
$\mathcal{L}, \mathcal{R}$ & linear operators
\end{tabular}

The material is based upon work supported by the European Office of Aerospace Research and Development, Air Force Research Laboratory, Air Force Office of Scientific Research, under Grants No. F49620-02-1-0362 (Caltech) and FA8655-03-1-3059 ( $\mathrm{nu}$ modelling S.L.) monitored by Dr. John D. Schmisseur (AFOSR) and Mr. Wayne Donaldson (EOARD).

\author{
Greek symbols \\ $\alpha \quad$ real wavenumber in the $x$-direction \\ $\beta \quad$ real wavenumber in the $z$-direction \\ $\varepsilon \quad$ infinitesimal quantity \\ $\kappa \quad$ ratio of specific heats \\ $\mu \quad$ viscosity \\ $\Omega \quad$ complex eigenvalue
}

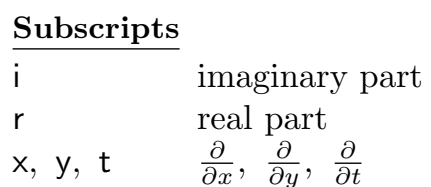




\section{Introduction}

Our present concern is with linear stability analysis of compressible open cavity flows. The relative complexity of the geometry has resulted in a large amount of information being available on one particular aspect of this stability problem, namely that of the shear-layer emanating from the upstream corner of the cavity. In real cavity flows this shear layer has significant non-local interactions, in the form of scattering into acoustic waves at the downstream edge, and in turn the receptivity of the separated shear layer to these acoustic waves. In most previous work the shear layer is seen in isolation from the cavity and treated as an one-dimensional profile whose stability characteristics are then semi-empirically correlated with the geometric features of the cavity in order to arrive at the celebrated Rossiter formula which predicts resonance frequencies as a function of the flow Mach number. Recently Alvarez et al. ${ }^{1}$ have made progress in developing improved linear models of the scattering/receptivity, and acoustic propagation aspects of the Rossiter modes and are able to eliminate many of the empirical parameters in the original Rossiter model.

However, there are also significant interactions between the shear layer and the flow within the cavity. In the most extreme form, this leads to a completely different form of cavity resonance-the so-called wake mode. ${ }^{6,13}$ The wake mode appears to be a global instability more akin to vortex shedding from bluff bodies; unlike the Rossiter mode the frequency of oscillation is independent of Mach number indicating that acoustic feedback does not play a role in the instability. However, wake mode is not commonly observed in shallow cavity flows at moderate subsonic to supersonic Mach number. It is known, for example, that the development of three-dimensionality in the resonant oscillations plays a role in suppressing the wake mode instability. ${ }^{7,14}$

Between the two extremes (Rossiter and wake mode) are a large number of experimental observations that involve significant interactions between the shear layer dynamics and the flow within the cavity (e.g. ${ }^{5,8}$ ). However, there is very little theory available with which to understand the interaction and its potential impact on quantities of interest such as frequency and amplitude of oscillations, the interactions of multiple tones, loads on internal cavity surfaces, etc.

Our work is aimed at calculating and understanding the instabilities and resonance of open cavities over the entire parameter space, including those regimes where significant shear layer and cavity interactions occur. In order to do this, we must compute the eigenspectrum of the cavity steady flow field which is inhomogeneous in at least two (streamwise and depth) spatial directions (in future work we will also consider cavities that are inhomogeneous in the third, spanwise direction).
Advances in algorithms and the sustained increase in hardware capabilities have permitted the development of novel tools to probe into the (global) eigenspectrum of such flows. In our previous work ${ }^{20}$ we made some preliminary calculations using an existing database of two-dimensional DNS computations of cavities with laminar upstream boundary layers. These calculations show clearly that the shear-layer instabilities are but one feature of a global cavity instability mode that involves interaction with the internal cavity flow. The methodology we used extracted the global instability modes from time series of the residuals of a sub-critical state. In the present paper, we extend this methodology to direct solution of the two-dimensional eigenvalue problem (EVP) for compressible flow.

The remainder of the paper is organized as follows. In the next section we present the equations for the linear stability of compressible flows with two inhomogeneous directions. In the following section we describe our numerical method for solving the 2D EVP. Results are given beginning with cases that serve to validate the numerical method and illustrate our approach. These include an incompressible square duct, the flat plate boundary layer from incompressible speed to $M=6$, and an isolated parallel two-dimensional shear layer. Finally, preliminary results for the open cavity flow are presented.

\section{Linear Stability Theory}

The analysis of flow stability is based on the compressible equations of motion

$$
\begin{aligned}
\frac{\partial \rho}{\partial t}+\nabla \cdot(\rho \mathbf{u})= & 0 \\
\frac{\partial(\rho \mathbf{u})}{\partial t}+\nabla \cdot(\rho \mathbf{u u})= & \frac{-1}{\gamma M^{2}} \nabla p \\
& +\frac{1}{R e} \nabla \cdot \sigma, \\
\frac{\partial p}{\partial t}+\mathbf{u} \cdot \nabla p+\gamma p \nabla \cdot \mathbf{u}= & \frac{\gamma}{\operatorname{RePr} \nabla \cdot(\kappa \nabla T)} \\
& +\frac{\gamma(\gamma-1) M^{2}}{\operatorname{Re}} \Phi,
\end{aligned}
$$

where,

$$
\sigma=\mu\left[\left(\nabla \mathbf{u}+\nabla \mathbf{u}^{T}\right)-\frac{2}{3}(\nabla \cdot \mathbf{u}) \mathbf{I}\right]
$$

and

$$
\Phi=\frac{1}{2}\left(\nabla \mathbf{u}+\nabla \mathbf{u}^{T}\right): \sigma
$$

are the viscous stress tensor and the dissipation function, respectively, and Stokes' hypothesis has been invoked. Here $\mu$ and $\kappa$ are nondimensionalized by their free-stream values (which appear in the definitions of $R e$ and $P r$, respectively, and pressure is normalized 
with the free-stream density and velocity. The equation of state of perfect gases with constant $\gamma=1.4$ has been used. We take $\operatorname{Pr}=0.7$.

Central to linear flow instability research is the concept of decomposition of any flow quantity into an $O(1)$ steady or time-periodic laminar basic flow upon which small-amplitude three-dimensional disturbances are permitted to develop. The most general framework in which a linear instability analysis can be performed is one in which three inhomogeneous spatial directions are resolved and time-periodic small-amplitude disturbances, inhomogeneous in all three directions, are superimposed upon the underlying steady or time-periodic $O(1)$ basic state. The related three-dimensional global TriGlobal instability Ansatz (named according to the dimensionality of the basic state ${ }^{18,19}$ ) yields a three-dimensional eigenvalue problem in which all three spatial directions must be resolved simultaneously in a coupled manner. Though this most general Ansatz is consistent with the separability in the governing equations of time on the one hand and the three spatial directions on the other, the size of the resulting EVP is such that currently available computing hardware and algorithms permit its solution in a very limited range of Reynolds numbers, of $R e \approx O\left(10^{2}\right)$.

\section{BiGlobal linear theory:}

The compressible two-dimensional linear EVP

In order to proceed, the basic state is assumed independent of one spatial coordinate, $z$, an assumption in line with the two-dimensional cavity geometry. ${ }^{13}$ Flow quantities are then decomposed according to

$$
\mathbf{q}(x, y, z, t)=\overline{\mathbf{q}}(x, y)+\varepsilon \tilde{\mathbf{q}}(x, y, z, t),
$$

with $\overline{\mathbf{q}}=(\bar{u}, \bar{v}, \bar{w}, \bar{T}, \bar{p})^{\mathrm{T}}$ and $\tilde{\mathbf{q}}=(\tilde{u}, \tilde{v}, \tilde{w}, \tilde{T}, \tilde{p})^{\mathrm{T}} \operatorname{rep}-$ resenting the steady two-dimensional basic flow and the unsteady three-dimensional infinitesimal perturbations, respectively, the latter being inhomogeneous in $x$ and $y$ and periodic in $z$. Note also that, unlike the incompressible case, pressure is a predictive variable in, rather than a constraint of, the equations of motion. On substituting (4) into the governing equations (1-3), taking $\varepsilon \ll 1$ and linearizing about $\overline{\mathbf{q}}$, one may write

$$
\tilde{\mathbf{q}}(x, y, z, t)=\hat{\mathbf{q}}(x, y) \mathrm{e}^{\mathrm{i} \Theta_{2 \mathrm{D}}}+c . c .,
$$

with $\hat{\mathbf{q}}=(\hat{u}, \hat{v}, \hat{w}, \hat{\theta}, \hat{p})^{\mathrm{T}}$ representing the vector of twodimensional complex amplitude functions of the infinitesimal three-dimensional perturbations, $\Omega$ a complex eigenvalue and

$$
\Theta_{2 \mathrm{D}}=\beta z-\Omega t
$$

a complex phase function.

The linear disturbance equations of BiGlobal stability analysis are obtained at $O(\varepsilon)$ by substituting the decomposition (4-6) into the equations of motion, subtracting out the $O(1)$ basic flow terms and neglecting terms at $O\left(\varepsilon^{2}\right)$. In the present temporal framework, $\beta$ is taken to be a real wavenumber parameter describing an eigenmode in the $z$-direction, while the complex eigenvalue $\Omega$, and the associated eigenvectors $\hat{\mathbf{q}}$ are sought. The real part of the eigenvalue, $\Omega_{\mathrm{r}} \equiv \Re\{\Omega\}$, is related with the frequency of the global eigenmode while the imaginary part is its growth/damping rate; a positive value of $\Omega_{\mathrm{i}} \equiv \Im\{\Omega\}$ indicates exponential growth of the instability mode $\tilde{\mathbf{q}}=\hat{\mathbf{q}} \mathrm{e}^{\mathrm{i} \Theta_{2 \mathrm{D}}}$ in time $t$ while $\Omega_{\mathrm{i}}<0$ denotes decay of $\tilde{\mathbf{q}}$ in time. The system for the determination of the eigenvalue $\Omega$ and the associated eigenfunctions $\hat{\mathbf{q}}$ in its most general form can be written as the complex non-symmetric generalized EVP

$$
\mathcal{L} \hat{\mathbf{q}}=\Omega \mathcal{R} \hat{\mathbf{q}}
$$

or, more explicitly,

$$
\begin{aligned}
& \left(\begin{array}{ccccc}
\mathcal{L}_{x \hat{u}} & \mathcal{L}_{x \hat{v}} & \mathcal{L}_{x \hat{w}} & \mathcal{L}_{x \hat{\theta}} & \mathcal{I L}_{x \hat{p}} \\
\mathcal{L}_{y \hat{u}} & \mathcal{L}_{y \hat{v}} & \mathcal{L}_{y \hat{w}} & \mathcal{L}_{y \hat{\theta}} & \mathcal{I} \mathcal{L}_{y \hat{p}} \\
\mathcal{L}_{z \hat{u}} & \mathcal{L}_{z \hat{v}} & \mathcal{L}_{z \hat{w}} & \mathcal{L}_{z \hat{\theta}} & \mathcal{I L}_{z \hat{p}} \\
\mathcal{L}_{e \hat{u}} & \mathcal{L}_{e \hat{v}} & \mathcal{L}_{e \hat{w}} & \mathcal{L}_{e \hat{\theta}} & \mathcal{I} \mathcal{L}_{e \hat{p}} \\
\mathcal{J} \mathcal{L}_{c \hat{u}} & \mathcal{J} \mathcal{L}_{c \hat{v}} & \mathcal{J} \mathcal{L}_{c \hat{w}} & \mathcal{J} \mathcal{L}_{c \hat{\theta}} & \mathcal{L}_{c \hat{p}}^{G}
\end{array}\right)\left(\begin{array}{c}
\hat{u} \\
\hat{v} \\
\hat{w} \\
\hat{\theta} \\
\hat{p}
\end{array}\right) \\
& =\Omega\left(\begin{array}{ccccc}
\mathcal{R}_{x \hat{u}} & 0 & 0 & 0 & 0 \\
0 & \mathcal{R}_{y \hat{v}} & 0 & 0 & 0 \\
0 & 0 & \mathcal{R}_{z \hat{w}} & 0 & 0 \\
0 & 0 & 0 & 0 & \mathcal{I R}_{e \hat{p}} \\
0 & 0 & 0 & \mathcal{J R}_{c \hat{\theta}} & \mathcal{R}_{c \hat{p}}^{G}
\end{array}\right)\left(\begin{array}{c}
\hat{u} \\
\hat{v} \\
\hat{w} \\
\hat{\theta} \\
\hat{p}
\end{array}\right),
\end{aligned}
$$

subject to appropriate boundary conditions. Here the linearized equation of state

$$
\hat{p}=\hat{\rho} / \bar{\rho}+\hat{\theta} / \bar{T}
$$

has been used, viscosity and thermal conductivity of the medium have been taken as functions of temperature alone, resulting in

$$
\hat{\mu}=\frac{d \bar{\mu}}{d T} \hat{\theta}, \hat{\kappa}=\frac{d \bar{\kappa}}{d T} \hat{\theta} .
$$

Moreover,

$$
\mathcal{I}=I_{G L}^{G}, \mathcal{J}=I_{G}^{G L}
$$

are interpolation arrays transferring data from the Gauss-Lobatto to the Gauss and from the Gauss to the Gauss-Lobatto spectral collocation grids, respectively, and will be defined in the next section. The entries of matrix $\mathcal{L}$ are presented in the Appendix; all sub-matrices are defined on a two-dimensional Chebyshev Gauss-Lobatto (CGL) grid, except for $\mathcal{L}_{c \hat{p}}^{G}$ and $\mathcal{R}_{c \hat{p}}^{G}$, which are defined on a two-dimensional Chebyshev Gauss (CG) grid.

\section{Classic linear theory:}

\section{The one-dimensional compressible linear EVP}

When developing a new algorithm for the solution of (7) it is convenient to compare results against those 
of the established classic theory of linear instability of boundary- and shear-layer flows (cf. Mack ${ }^{11}$ ). The latter theory is based on the Ansatz

$$
\mathbf{q}(x, y, z, t)=\overline{\mathbf{q}}(y)+\varepsilon \hat{\mathbf{q}}(y) \mathrm{e}^{\mathrm{i} \Theta_{1 \mathrm{D}}}+c . c .
$$

In (9) $\hat{\mathbf{q}}$ is the vector of one-dimensional complex amplitude functions of the infinitesimal perturbations and $\Omega$ is in general complex. The phase function, $\Theta_{1 \mathrm{D}}$, is

$$
\Theta_{1 \mathrm{D}}=\alpha x+\beta z-\Omega t
$$

where $\alpha$ and $\beta$ are wavenumber parameters in the spatial directions $x$ and $z$, respectively.

Substitution of the decomposition (9-10) into the governing equations (1-3) linearization and consideration of terms at $O(\varepsilon)$ results in the eigenvalue problem governing linear stability of boundary- and shear-layer flows; the same system results directly from (7) if one makes the following ("parallel flow") assumptions:

- $\partial \bar{q} / \partial x \equiv 0, \partial \bar{q} / \partial x \equiv 0$

(basic flow independent of $x$ ),

i.e. $\partial \hat{q} / \partial x \equiv \mathrm{i} \alpha \hat{q}, \partial \hat{q} / \partial z \equiv \mathrm{i} \beta \hat{q}$

(harmonic expansion of disturbances in $x$ and $z$ ),

- $\bar{v} \equiv 0$, and

- $\bar{p} \equiv$ cnst.

then (7) takes the form of the system of equations governing linear stability of viscous compressible boundary- and shear-layer flows (cf. eqns. (8.9) of $\left.\mathrm{Mack}^{11}\right)$. This provides direct means for comparisons between the present novel and the established methodologies. It should be noted that the crucial difference between the two-dimensional eigenvalue problem (7) and the limiting case of the one-dimensional $\mathrm{EVP}$ is that the eigenvector $\hat{q}$ in (7) comprises twodimensional amplitude functions, while those in the limiting parallel-flow case are one-dimensional. Further, while $\bar{p}(y)=$ cnst. in taken to be a constant in one-dimensional basic states satisfying $(9), \bar{p}(x, y)$ appearing in (7) is, in general, a known function of the two resolved spatial coordinates.

\section{Solution approach}

It has been stressed that a key difference between the classic linear stability theory and the present BiGlobal analysis methodology is that the amplitude functions of the small-amplitude disturbances in the latter framework develop along two inhomogeneous spatial directions which must be solved simultaneously. Consequently, the resolution requirements for adequate description of BiGlobal instabilities can be quite challenging; a thorough discussion of this point, mainly focusing on incompressible flows, has been presented by Theofilis. ${ }^{19}$ The demands placed on memory by a compressible BiGlobal analysis are aggravated not only by the need to solve the energy equation in addition to those of incompressible flow, but also by the well-known fact that increasing Mach numbers result in tighter eigenmode structures to be resolved, compared with their incompressible counterparts. Consequently, numerical methods of high resolution capacity are essential in this problem.

In the present analysis spectral collocation has been used, based on two sets of Chebyshev points, the Chebyshev Gauss-Lobatto (CGL)

$$
\xi_{j}=\cos \frac{j \pi}{N}, \quad(j=0, \ldots, N),
$$

the extrema of the $N$-th order Chebyshev polynomials $T_{N}(\xi)=\cos \left(N \cos ^{-1} \xi\right)$, and the Chebyshev Gauss (CG)

$$
\xi_{j}=\cos \frac{\pi(2 j+1)}{2 N}, \quad(j=0, \ldots, N-1),
$$

the roots of the $T_{N}(\xi)$; both sets of points are depicted in figure 1 . These two sets of points are introduced in view of the different type of boundary conditions pertaining to the stability problem. While boundary conditions are readily available for the disturbance velocity components and temperature perturbations, no physically meaningful boundary conditions exist for the pressure perturbation. Consequently the momentum and energy equations are collocated on the CGL points, while the equation of continuity is collocated on the CG points of a twodimensional staggered grid.

Each sub-matrix of the linear two-dimensional eigenvalue problem (7) has the form

$$
\mathcal{L}=A \partial_{x x}^{2}+B \partial_{x y}^{2}+C \partial_{y y}^{2}+D \partial_{x}+E \partial_{y}+F
$$

and is either defined on the CGL or the CG points. First derivatives on the CGL points are calculated using the collocation derivative matrix, presented by Boyd, ${ }^{3}$

$$
\left(D_{G L}^{(1)}\right)_{k, j}= \begin{cases}\frac{2 N^{2}+1}{6}, & j=k=0 \\ -\frac{\xi_{j}}{2\left(1-\xi_{j}^{2}\right)}, & j=k \\ \frac{\bar{c}_{k}}{\bar{c}_{j}} \frac{(-1)^{j+k}}{\xi_{k}-\xi_{j}}, & j \neq k \\ -\frac{2 N^{2}+1}{6}, & j=k=N\end{cases}
$$

with $\xi_{j}$ defined by (11), while those on the CG points are calculated using 


$$
\left(D_{G}^{(1)}\right)_{k, j}= \begin{cases}\frac{0.5 \xi_{j}}{1-\xi_{j}^{2}}, & j=k \\ \frac{(-1)^{k+j}}{\xi_{k}-\xi_{j}} \sqrt{\frac{1-\xi_{j}^{2}}{1-\xi_{k}^{2}}}, & j \neq k\end{cases}
$$

with $\xi_{j}$ defined by (12). Higher derivatives on either the CGL or the CG points may be calculated using

$$
D_{k, j}^{(m)}=\left(D_{k, j}^{(1)}\right)^{m}
$$

while the cross-derivatives appearing in (7) may be calculated using

$$
\partial_{x y}^{2} f_{i, k}=\sum_{l} D_{k, l}^{y}\left[\sum_{j} D_{i, j}^{x} f_{j, l}\right]
$$

with $(k, l=0, \cdots, N y),(i, j=0, \cdots, N x)$ and superscripts denote differentiation along the indicated coordinate.

Data may be transferred between the grids using the interpolation arrays $I_{G L}^{G}$ and $I_{G}^{G L}$ introduced in (7),

$$
\begin{aligned}
I_{G L}^{G} & =C_{G}^{-1} C_{G L}, \\
I_{G}^{G L} & =C_{G L}^{-1} C_{G},
\end{aligned}
$$

where, for $(k=0, \cdots, N)$,

$$
\begin{aligned}
\left(C_{G L}\right)_{k, j} & =\frac{2}{c_{k} c_{j}} \cos \frac{k j \pi}{N}, \quad(j=0, \cdots, N) \\
\left(C_{G L}\right)_{j, k}^{-1} & =\cos \frac{k j \pi}{N}, \quad(j=0, \cdots, N) \\
\left(C_{G}\right)_{k, j} & =\frac{2}{N c_{k}} \cos \frac{\left(j+\frac{1}{2}\right) \pi k}{N},(j=0, \cdots, N-1) \\
\left(C_{G}\right)_{j, k}^{-1} & =\cos \frac{\left(j+\frac{1}{2}\right) \pi k}{N},(j=0, \cdots, N-1),
\end{aligned}
$$

$c_{0}=c_{N}=2$ and $c_{k, j}=1,(k, j=1, \cdots, N-1)$.

Flexibility is added into the solution algorithm by incorporating different types of coordinate transformations, to be employed independently in each of the resolved $x$ - and $y$-directions. The mappings and the related transformation metrics used in what follows are, that due to Kosloff and Tal-Ezer, ${ }^{9}$ which maps the standard CGL domain $[-1,1]$ onto itself through

$$
\begin{aligned}
x & =\frac{\sin ^{-1}(p \xi)}{\sin ^{-1} p} \\
\frac{d \xi}{d x} & =\frac{\sin ^{-1} p}{p} \sqrt{1-(p \xi)^{2}}
\end{aligned}
$$

a well-tested mapping transformation for semiinfinite domains of boundary-layer type ${ }^{22}$

$$
\begin{aligned}
x & =l \frac{1-\xi}{1+s+\xi}, \\
\frac{d \xi}{d x} & =-l \frac{2+s}{(\xi+l)^{2}}
\end{aligned}
$$
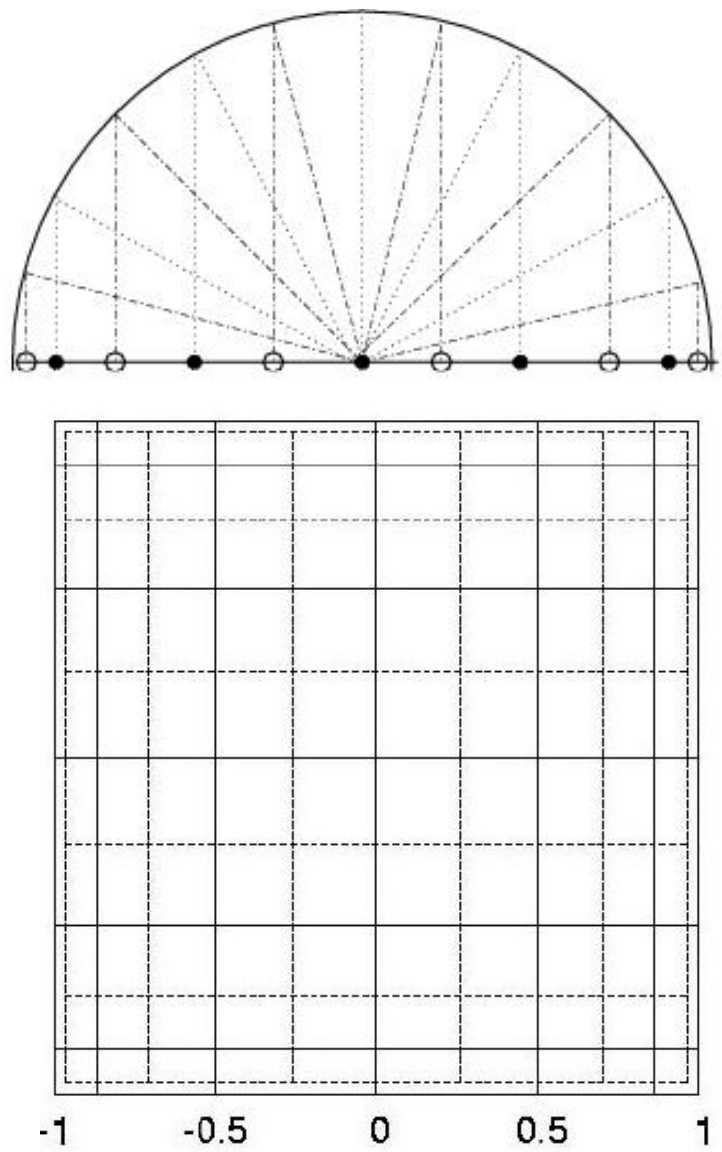

Fig. 1 Upper: The CGL (full symbols) and the CG (open symbols) collocation points. Lower: A two-dimensional staggered grid after mapping the two sets of collocation points using the Kosloff Tal-Ezer transformation

as well as a transformation of $[-1,1]$ onto $(-\infty, \infty)$,

$$
\begin{aligned}
x & =l \frac{\sinh (s \xi)}{\sinh s}, \\
\frac{d \xi}{d x} & =\frac{\sinh s}{l s} \sqrt{1+\left(\frac{x}{l}\right)^{2} \sinh ^{2} s}
\end{aligned}
$$

The solution domain resulting by employing the Kosloff and Tal-Ezer transformation in both the $x$ and $y$ directions is also shown in figure 1.

Using the tools exposed the compressible BiGlobal linear eigenvalue problem (7) is transformed into a discrete matrix eigenvalue problem. In the absence of prior information on interesting regions of the parameter space, the latter problem is solved using the QZ algorithm.

\section{Results}

\section{The rectangular duct}

Our first concern, when developing a new algorithm has been with the recovery of established linear stability results in a variety of flows related with the 


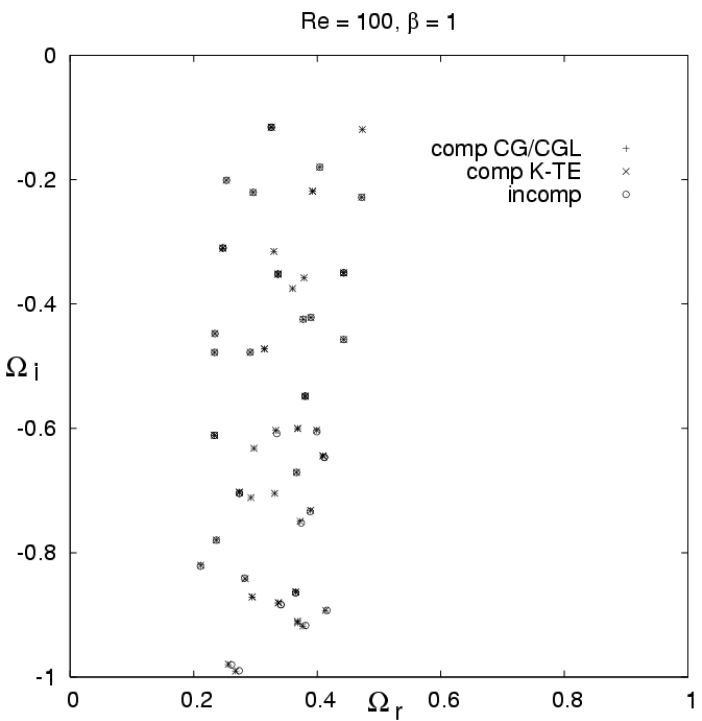

Fig. 2 The eigenspectrum of rectangular duct flow, obtained using the present analysis methodology at $M=10^{-4}$, and compared with incompressible theory.

open cavity problem. In view of the virtual absence of analytically known two-dimensional compressible basic states, the incompressible rectangular duct, ${ }^{15}$ for which an analytic solution is known to exist, ${ }^{12,16}$ has been first analyzed at $M=10^{-4}$. Figure 2 shows results obtained by numerical solution of (7) at $R e=100, \beta=1$, using either an unmapped square domain or one in which the Kosloff-Tal Ezer ${ }^{9}$ (KTE) mapping is incorporated. Superimposed are results obtained using an independent algorithm for the solution of the incompressible BiGlobal stability problem, ${ }^{16,21}$ based on Krylov subspace iteration for the recovery of the most significant part of the eigenspectrum. Excellent agreement is obtained between all modes delivered by the incompressible analysis and either compressible approach. In addition, the present solution of the compressible EVP delivers modes related with the energy equation which, at the conditions examined, are slightly more stable than the modes known from incompressible analysis.

\section{The flat-plate boundary layer}

This flow been the subject of extensive numerical investigation by $\mathrm{Mack}^{11}$ in a series of papers spanning more than three decades. Viscous instability dominates at the incompressible and low subsonic limit, but the development of inflection points as the Mach number increases results in the presence of a multitude (in fact infinite) instability modes, both viscous and inviscid in nature. The basic state, describing zero-pressure-gradient compressible laminar boundary layer flow over a flat plate, as well as an approach for the numerical solution of this problem are presented by Mack. ${ }^{11}$ For completeness we present the equations solved in dimensionless form

$$
\begin{aligned}
\frac{d}{d \eta}\left(\frac{d \bar{u}}{d \eta}\right)+\frac{g}{\bar{\mu}}\left(\bar{\mu} \frac{d \bar{u}}{d \eta}\right) & =0, \\
\frac{d}{d \eta}\left(\frac{\bar{\mu}}{\operatorname{Pr}} \frac{d \bar{T}}{d \eta}\right)+\frac{g P r}{\bar{\mu}}\left(\frac{\bar{\mu}}{\operatorname{Pr}} \frac{d \bar{T}}{d \eta}\right) & \left.=-2 \bar{\mu}\left(\frac{d \bar{u}}{d \eta}\right)^{2} 23\right)
\end{aligned}
$$

where $\eta$ is the boundary-layer similarity variable and use has been made of

$$
g(\eta)=\frac{1}{2} \int_{0}^{\eta} \frac{\bar{u}}{\bar{T}} d \eta
$$

The relations

$$
R=\sqrt{R_{x}}
$$

and

$$
R=\left(1 / \delta^{*}\right) R_{\delta^{*}}
$$

relate the Reynolds number $R$ built on an integral length scale $L^{*}$ and that built on the dimensional streamwise coordinate $x^{*}$ and the boundary layer displacement thickness $\delta^{*}$ respectively. A linear (Chapman) viscosity law has been used at $T_{e}=288.89$ and the boundary-layer coordinate transformation presented earlier is employed for the spectral collocation solution of this problem.

Several test cases have been solved, first under the parallel flow assumption. Some results for the computed eigenvalues of the unstable mode are presented next. Eigenfunctions follow in graphical form in figures 3-4.

- Almost incompressible Case I:

$M=10^{-3}, R=800, \alpha=0.1200$,

$\psi=\tan ^{-1}(\beta / \alpha)=30, \delta^{*}=1.720788 L$.

Computed Eigenvalue:

$\omega=\omega_{r}+i \omega_{i}=0.040583234+0.001728706 i$.

- Compressible Case II:

$M=1, R=1000, \alpha=0.1$,

$\psi=45, \delta^{*}=2.004500 L$.

Computed Eigenvalue:

$\omega=\omega_{r}+i \omega_{i}=0.038017729+0.001350618 i$.

- High supersonic Case III:

$M=6, R=1000, \alpha=0.0800$,

$\psi=45, \delta^{*}=17.696682 \mathrm{~L}$.

Computed Eigenvalue:

$\omega=\omega_{r}+i \omega_{i}=0.072487839+0.001052149 i$.

The resolution requirements for compressible linear stability analysis become clear by inspection of the eigenmode results. As the Mach number increases, sharp gradients develop both in the near-wall region and the neighborhood of the critical layer, the latter moving well into the free-stream.

Next, the dependence of the basic state on $x$ is reinstated and the two-dimensional EVP is solved, using 

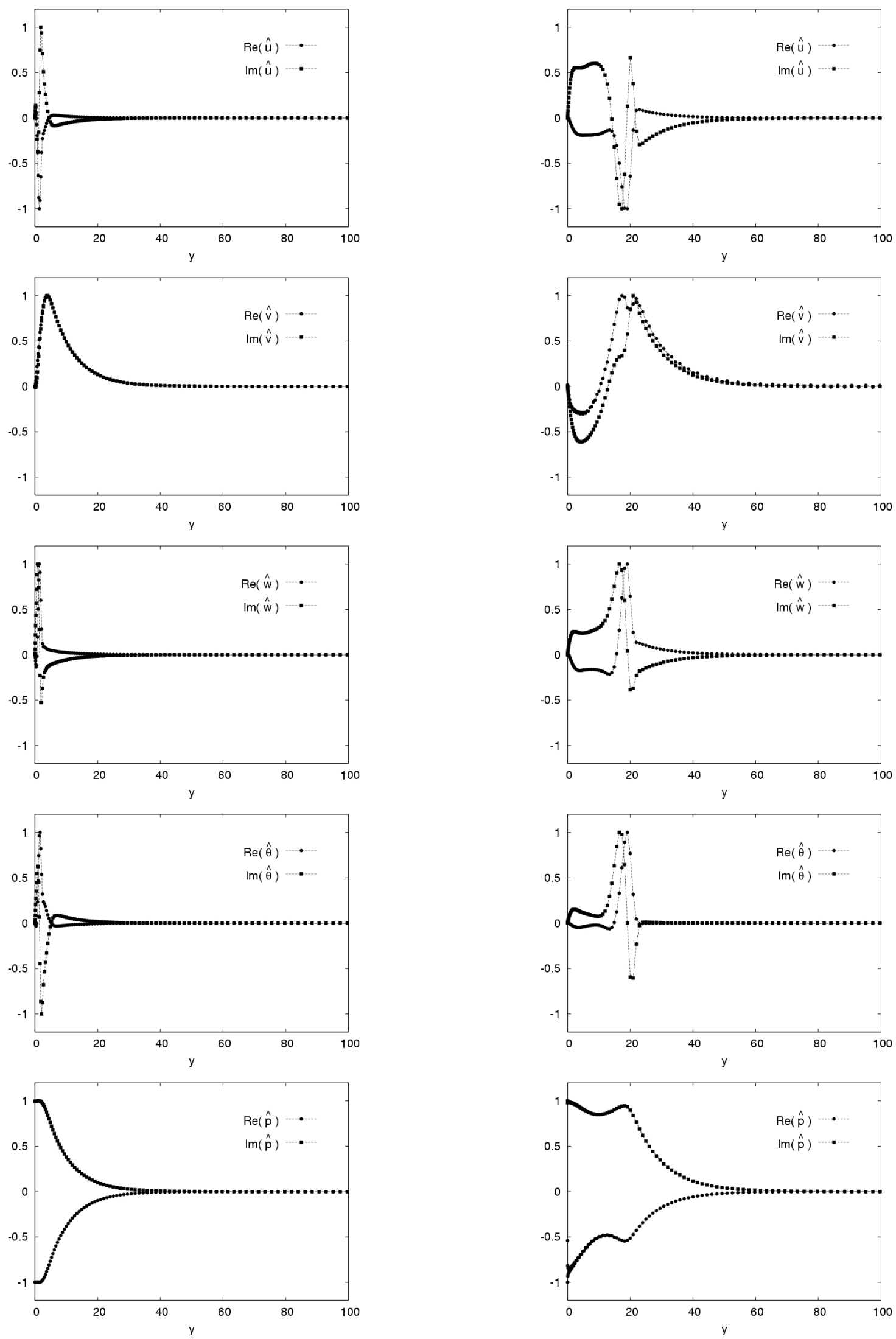

Fig. 3 The most unstable eigenvector of compressible flat-plate flow at $M a=1, R=$ $1000, \alpha=\beta=0.1$

Fig. 4 The most unstable eigenvector of compressible flat-plate flow at $M a=6, R=$ $1000, \alpha=\beta=0.08$ 
the one-dimensional base flow profiles delivered by (22-23). No direct comparisons with the classic linear theory are possible in this case, because of the slow but finite growth of the basic state in the streamwise coordinate. An approximation of the results delivered under the simplifying assumptions may be obtained by imposing on the two-dimensional amplitude functions a wave-like character behavior through the boundary conditions

$$
\frac{\partial^{2} \hat{q}(x, y)}{\partial x^{2}}+\tilde{\alpha}^{2} \hat{q}(x, y)=0
$$

imposed at both the inflow and the outflow boundaries, with $\tilde{\alpha}=2 \pi / L_{x}$ determined via the streamwise extent $L_{x}$ of the integration domain. Results obtained exhibit qualitative analogous behavior along the wellresolved $y$ direction, while the boundary condition (24) selects amplitude functions with wave-like character in $x$. Nevertheless, in a manner analogous with incompressible flow, ${ }^{19}$ the key finding in this part of the validation studies is that the memory requirements for compressible BiGlobal instability analysis at high (coupled) resolution can be very demanding. Currently parallelization of the numerical solution of (7) is investigated.

\section{A shear layer}

The final validation case, relevant to compressible open cavity flow, is that of a model shear-layer, the basic state of which is given by

$$
\bar{u}=\tanh (y), \quad y \in(-\infty, \infty) .
$$

The temperature distribution is obtained by the Crocco-Busemann integral

$$
\begin{aligned}
\bar{T} & =\frac{1}{2}(\gamma-1) M_{\infty}^{2}\left[1-\tanh ^{2}(y)\right] \\
& +\frac{1}{2}[1+\tanh (y)]+\frac{1}{2}[1-\tanh (y)] .
\end{aligned}
$$

Again, in view of the one-dimensional base flow profile (26-27), classic linear theory is used, albeit solved as a limiting case of (7). We first compare our results to those of Blumen ${ }^{2}$ and Macaraeg et al. ${ }^{10}$ for subsonic Mach numbers. Since Blumen's theory is valid at the inviscid limit we set, as was done in, ${ }^{10}$ the Reynolds number $R=10000$ throughout our calculations. The comparisons may be found in table 1. At finite Reynolds numbers sample results for the growth rate of the unstable (stationary) modes may be found in table 2 .

While successful in identifying elements of instabilities in the open cavity flow, the single-domain algorithm discussed cannot be extended to cover the entire domain of this flow. In order to extend the BiGlobal

\begin{tabular}{ccccc}
\hline & & & $\omega_{i}$ & \\
$M$ & $\alpha$ & Blumen $^{2}$ & Macaraeg et al. & present \\
\hline 0.0 & 0.445 & 0.190 & 0.18954 & 0.189539 \\
0.2 & 0.426 & 0.181 & 0.18112 & 0.181156 \\
0.4 & 0.409 & 0.158 & 0.15760 & 0.157581 \\
0.6 & 0.370 & 0.122 & 0.12180 & 0.121763 \\
0.8 & 0.279 & 0.078 & 0.07760 & 0.077607 \\
\hline
\end{tabular}

Table 1 Comparison of present results with those of earlier inviscid and viscous analyses.

\begin{tabular}{rccc}
\hline$R e$ & $M$ & $\alpha$ & $\omega_{i}$ \\
\hline $2 \times 10^{2}$ & 0.2 & 0.426 & 0.173858 \\
$10^{3}$ & 0.2 & 0.426 & 0.179787 \\
$10^{4}$ & 0.2 & 0.426 & 0.181156 \\
$2 \times 10^{2}$ & 0.6 & 0.370 & 0.114727 \\
$10^{3}$ & 0.6 & 0.370 & 0.120443 \\
$10^{4}$ & 0.6 & 0.370 & 0.121763 \\
\hline
\end{tabular}

Table 2 Effect of increasing $R e$ on the growth rates of shear layer instability.

stability analysis methodology to complex geometries with a certain degree of regularity, the spectral multidomain algorithm of Macaraeg et al. ${ }^{10}$ is an obvious candidate to be employed and investigations are currently underway in this direction.

\section{Compressible flow in an open cavity}

Turning to BiGlobal instability of compressible open cavity flow, we are first concerned with the issue of accurate basic states to be analyzed. Clearly only numerical solutions exist for the basic state; we compute them using DNS of the compressible, two-dimensional Navier-Stokes equations. Our methodology and validation are presented in detail in Colonius et al. ${ }^{13}$ The DNS computations are carried out over a range of parameters: $1<L / D<5$ (where $L$ is cavity length, and $D$ is depth), $20<L / \theta<120$ (where $\theta$ is the momentum thickness of the upstream laminar boundary layer at the cavity leading edge), $0.2<M<2.0$ (where $M$ is the Mach number), and $40<R e_{\theta}<90$. For supersonic (and high subsonic cases) a hybrid highorder Weighted Essentially Non-Oscillatory (WENO) / Compact finite difference scheme is used for shock capturing.

Earlier work has shown the potential that BiGlobal instability theory has in analyzing the instability of this class of flows (Theofilis and Colonius ${ }^{20}$ ). In the latter work, analysis of the time-signal provides a unified framework in which different known aspects of open cavity instability, such as the shear-layer and the acoustic modes, can be classified. Moreover, it was shown that modes akin to that of the lid-driven cavity flow ${ }^{17}$ exist in the two-dimensional open cavity. Up to now we have only considered the stability characteristics of the least damped eigenmode in a two- 


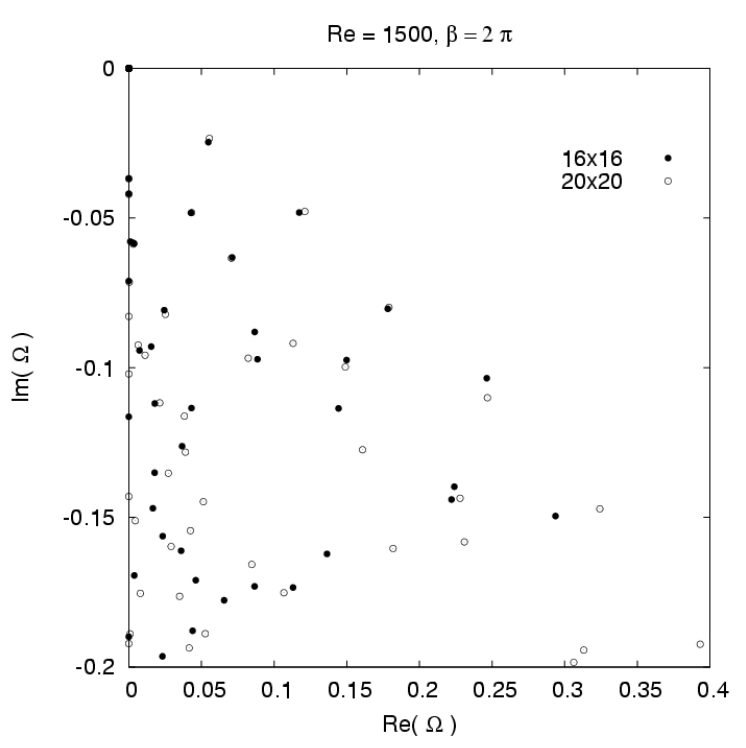

Fig. 5 The eigenspectrum of compressible open cavity flow at $R e=1500, M=0.325$ and $\beta=2 \pi$.

dimensional BiGlobal instability context, leaving the question of instability to three-dimensional BiGlobal eigenmodes open.

We present here preliminary computations of threedimensional modes using the new algorithm described in the previous sections. Based on previous work for the lid-driven cavity and flow over back step, we expect that the first three-dimensional bifurcation will be in the recirculating flow within the cavity. ${ }^{4,21}$ Thus for simplicity we only compute the eigenspectrum for the flow within the cavity proper. In the simplified analysis we apply a Neumann boundary condition for the velocity fluctuations along the top of the cavity. This is clearly a crude approximation that will be removed in future computations where the eigenspectra will be computed on a block rectangular grid that includes a the flow above the cavity. Thus we impose the boundary conditions:

$$
\hat{u}=\hat{v}=\hat{w}=0
$$

along the cavity surface

$$
\partial \hat{u} / \partial n=\partial \hat{v} / \partial n=\partial \hat{w} / \partial n=0,
$$

along the open cavity surface, and

$$
\partial \hat{\theta} / \partial n=\partial \hat{p} / \partial n=0,
$$

on all boundaries. Grid experimentation has been performed in order to reach convergence of the leastdamped portion of the eigenspectrum at a set of parameters $L / D=2, R e_{D}=1500$ (based on cavity depth and free-stream velocity) and $M=0.325$. Out of several values of the spanwise wavenumber $\beta$ examined, we present results for $\beta=2 \pi$, i.e. $L_{z}=|D|=1$.

Figure 5 shows the most interesting part of the eigenspectrum of the flow in question. At the flow parameters chosen a modest resolution of 20x20 collocation points per eigenmode, i.e. a leading dimension of 1000 of the matrices in the eigenvalue problem (7), suffices. Only damped traveling and stationary eigenmodes have been found within the range of spanwise wavenumbers $\beta$ examined. This implies that the steady solution from DNS persists in spanwise periodic three-dimensional domains corresponding up to the wavenumbers examined.

In figures (6-7) the spatial structure of the (linear) disturbance flow field is shown in terms of isocontours of $\hat{u}, \hat{v}, \hat{w}$ and the magnitude of the disturbance vorticity. Several aspects of these results, and analogous ones not presented here, may be stressed. First, it appears that there exists a connection of the modes inside the cavity with those in the oncoming flow, and that the present BiGlobal analysis is able to capture. Second, the significance of the downstream corner of the cavity, as known from experiment and numerical simulations, is evident in the amplitude functions of the leading eigenmodes. Third, these leading eigenmodes suggest possible means of three-dimensionalization of the steady basic flow once the respective modes become amplified.

\section{Conclusions}

A new methodology for the numerical solution of the compressible two-dimensional eigenvalue problem governing BiGlobal flow instability has been developed and validated against several canonical flows. It has been shown that spectral collocation methods on a two-dimensional staggered grid can deliver accurate results in an efficient manner. Preliminary investigations of the problem of BiGlobal instability of compressible flow in an aspect-ratio two open cavity flow have been performed. It was shown that three dimensional disturbances having wavenumbers in the range $\beta \in[0,2 \pi]$ are stable at for a particular cavity with $L / D=2$ and $M=0.325$. Further algorithmic developments are necessary to enable addressing the open cavity domain in its entirety, as well as to make computations of the BiGlobal eigenspectrum more efficient; once completed we will characterize the eigenspectrum of three dimensional disturbances more completely over a broad range of cavity parameters. 

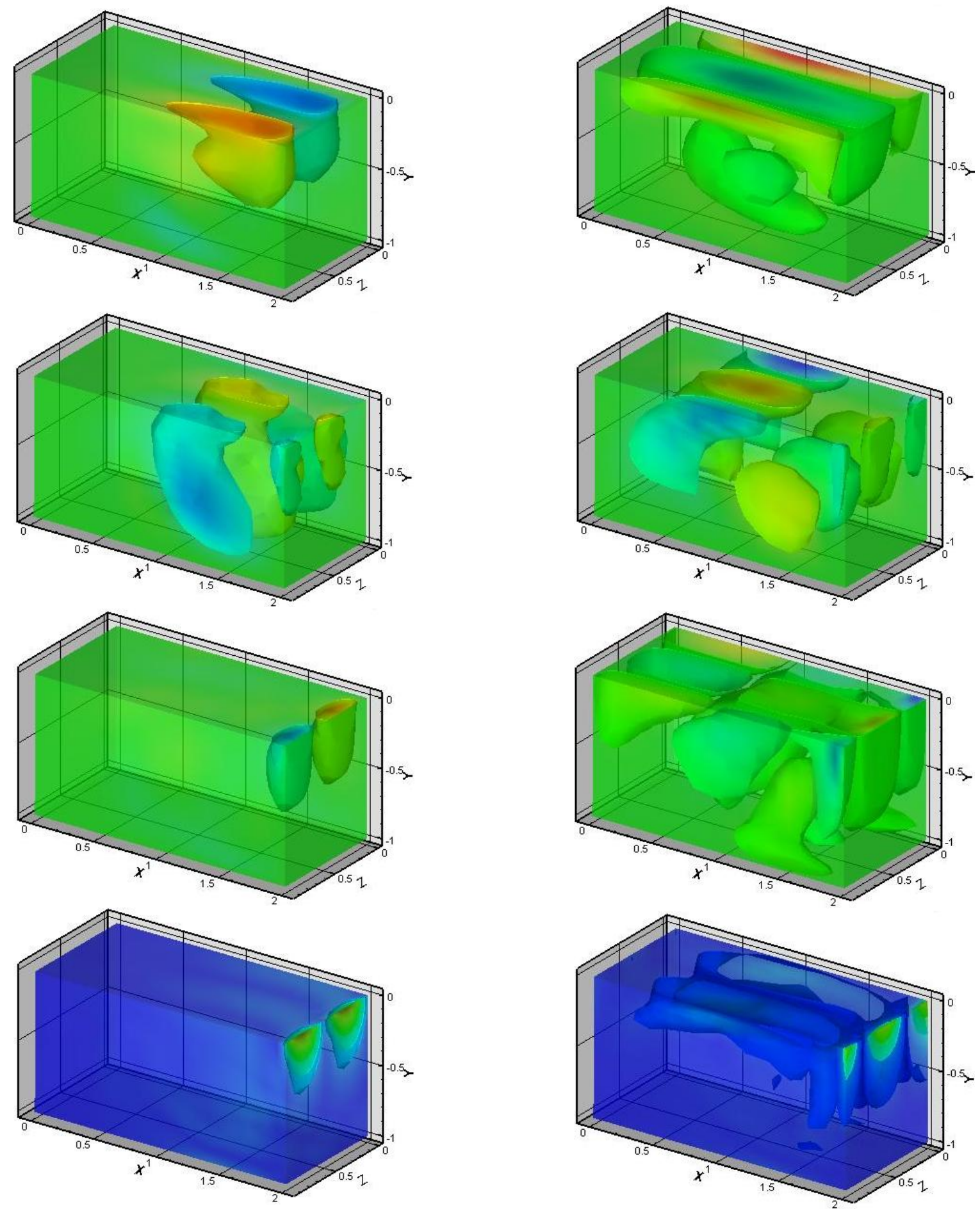

Fig. 6 Upper to lower: Isosurfaces of $\hat{u}, \hat{v}, \hat{w}$ and disturbance vorticity magnitude of the least stable traveling eigenmode of the flow.

Fig. 7 Upper to lower: Isosurfaces of $\hat{u}, \hat{v}, \hat{w}$ and disturbance vorticity magnitude of the least stable stationary eigenmode of the flow. 


\section{Appendix: The coefficients of the EVP}

$\underline{\text { Disturbance } x \text {-momentum: }}$

$$
\begin{aligned}
\mathcal{L}_{x \hat{u}}= & -\frac{4}{3} \frac{\bar{\mu}}{R e} \partial_{x x}^{2}+\left[-\frac{4}{3} \frac{1}{R e}\left(\frac{d \bar{\mu}}{d \bar{T}}\right) \bar{T}_{x}+\bar{\rho} \bar{u}\right] \partial_{x}-\frac{\bar{\mu}}{R e} \partial_{y y}^{2}+\left[-\frac{1}{R e}\left(\frac{d \bar{\mu}}{d \bar{T}}\right) \bar{T}_{y}+\bar{\rho} \bar{v}\right] \partial_{y} \\
& +\frac{\beta^{2} \bar{\mu}}{R e}+2 \bar{\rho} \bar{u}_{x}+\bar{\rho} \bar{v}_{y}+\mathrm{i} \beta \bar{\rho} \bar{w}+\bar{u} \bar{\rho}_{x}+\bar{v} \bar{\rho}_{y} \\
\mathcal{L}_{x \hat{v}}= & -\frac{1}{3} \frac{\bar{\mu}}{R e} \partial_{x y}^{2}-\frac{1}{R e}\left(\frac{d \bar{\mu}}{d \bar{T}}\right) \bar{T}_{y} \partial_{x}+\frac{2}{3} \frac{1}{R e}\left(\frac{d \bar{\mu}}{d \bar{T}}\right) \bar{T}_{x} \partial_{y}+\bar{\rho} \bar{u}_{y} \\
\mathcal{L}_{x \hat{w}}= & -\frac{\mathrm{i} \beta}{3 R e} \bar{\mu} \partial_{x}+\frac{2 \mathrm{i} \beta}{3 R e}\left(\frac{d \bar{\mu}}{d \bar{T}}\right) \bar{T}_{x} \\
\mathcal{L}_{x \hat{\theta}}= & -\frac{1}{R e}\left(\frac{d \bar{\mu}}{d \bar{T}}\right)\left(\frac{4}{3} \bar{u}_{x}-\frac{2}{3} \bar{v}_{y}\right) \partial_{x}-\frac{1}{R e}\left(\frac{d \bar{\mu}}{d \bar{T}}\right)\left(\bar{u}_{y}+\bar{v}_{x}\right) \partial_{y} \\
& -\frac{1}{R e}\left(\frac{d \bar{\mu}}{d \bar{T}}\right)\left(\mathrm{i} \beta \bar{w}_{x}+\frac{4}{3} \bar{u}_{x x}+\bar{u}_{y y}+\frac{1}{3} \bar{v}_{x y}\right)-\frac{1}{R e}\left(\frac{d^{2} \bar{\mu}}{d T^{2}}\right)\left(\frac{4}{3} \bar{T}_{x} \bar{u}_{x}+\bar{T}_{y} \bar{u}_{y}+\bar{T}_{y} \bar{v}_{x}-\frac{2}{3} \bar{T}_{x} \bar{v}_{y}\right) \\
& -\frac{\bar{\rho}}{T}\left(\bar{u} \bar{u}_{x}+\bar{v} \bar{u}_{y}\right) \\
\mathcal{L}_{x \hat{p}}= & \frac{1}{\gamma M^{2}} \partial_{x}+\frac{1}{\bar{T}}\left(\bar{u} \bar{u}_{x}+\bar{v} \bar{u}_{y}\right) \\
\mathcal{R}_{x \hat{u}}= & \mathrm{i} \bar{\rho}
\end{aligned}
$$

Disturbance $y$-momentum:

$$
\begin{aligned}
\mathcal{L}_{y \hat{u}}= & -\frac{1}{3} \frac{\bar{\mu}}{R e} \partial_{x y}^{2}+\frac{2}{3 R e}\left(\frac{d \bar{\mu}}{d T}\right) \bar{T}_{y} \partial_{x}-\frac{1}{R e}\left(\frac{d \bar{\mu}}{d T}\right) \bar{T}_{x} \partial_{y}+\bar{\rho} \bar{v}_{x} \\
\mathcal{L}_{y \hat{v}}= & -\frac{\bar{\mu}}{R e} \partial_{x x}^{2}+\left[-\frac{1}{R e}\left(\frac{d \bar{\mu}}{d \bar{T}}\right) \bar{T}_{x}+\bar{\rho} \bar{u}\right] \partial_{x}-\frac{4}{3} \frac{\bar{\mu}}{R e} \partial_{y y}^{2}+\left[-\frac{4}{3 R e}\left(\frac{d \bar{\mu}}{d \bar{T}}\right) \bar{T}_{y}+\bar{\rho} \bar{v}\right] \partial_{y} \\
& +\frac{\beta^{2} \bar{\mu}}{R e}+\bar{\rho} \bar{u}_{x}+2 \bar{\rho} \bar{v}_{y}+\mathrm{i} \beta \bar{\rho} \bar{w}+\bar{u} \bar{\rho}_{x}+\bar{v} \bar{\rho}_{y} \\
\mathcal{L}_{y \hat{w}}= & -\frac{\mathrm{i} \beta}{3} \frac{\bar{\mu}}{R e} \partial_{y}+\frac{2 \mathrm{i} \beta}{3} \frac{1}{R e}\left(\frac{d \bar{\mu}}{d T}\right) \bar{T}_{y} \\
\mathcal{L}_{y \hat{\theta}}= & -\frac{1}{R e}\left(\frac{d \bar{\mu}}{d \bar{T}}\right)\left(\bar{u}_{y}+\bar{v}_{x}\right) \partial_{x}-\frac{1}{R e}\left(\frac{d \bar{\mu}}{d \bar{T}}\right)\left(\frac{4}{3} \bar{v}_{y}-\frac{2}{3} \bar{u}_{x}\right) \partial_{y} \\
& -\frac{1}{R e}\left(\frac{d \bar{\mu}}{d \bar{T}}\right)\left(\mathrm{i} \beta \bar{w}_{y}+\frac{4}{3} \bar{v}_{y y}+\frac{1}{3} \bar{u}_{x y}+\bar{v}_{x x}\right)-\frac{1}{R e}\left(\frac{d^{2} \bar{\mu}}{d T^{2}}\right)\left(\bar{T}_{x} \bar{u}_{y}+\frac{4}{3} \bar{T}_{y} \bar{v}_{y}-\frac{2}{3} \bar{T}_{y} \bar{u}_{x}+\bar{T}_{x} \bar{v}_{x}\right) \\
& \left.-\frac{\bar{\rho}}{T}\left(\bar{u} \bar{v}_{x}+\bar{v} \bar{v}_{y}\right)\right] \\
\mathcal{L}_{y \hat{p}}= & \frac{1}{\gamma M^{2}} \partial_{y}+\frac{1}{T}\left(\bar{u} \bar{v}_{x}+\bar{v} \bar{v}_{y}\right) \\
\mathcal{R}_{y \hat{v}}= & \mathrm{i} \bar{\rho}
\end{aligned}
$$

\section{Disturbance $z$-momentum:}

$$
\begin{aligned}
\mathcal{L}_{z \hat{u}}= & -\frac{\mathrm{i} \beta}{3 R e} \bar{\mu} \partial_{x}-\frac{\mathrm{i} \beta}{R e}\left(\frac{d \bar{\mu}}{d \bar{T}}\right) \bar{T}_{x}+\bar{\rho} \bar{w}_{x} \\
\mathcal{L}_{z \hat{v}}= & -\frac{\mathrm{i} \beta}{3 R e} \bar{\mu} \partial_{y}-\frac{\mathrm{i} \beta}{R e}\left(\frac{d \bar{\mu}}{d \bar{T}}\right) \bar{T}_{y}+\bar{\rho} \bar{w}_{y} \\
\mathcal{L}_{z \hat{w}}= & -\frac{1}{R e} \bar{\mu} \partial_{x x}^{2}-\frac{1}{R e} \bar{\mu} \partial_{y y}^{2}+\left[-\frac{1}{R e}\left(\frac{d \bar{\mu}}{d \bar{T}}\right) \bar{T}_{x}+\bar{\rho} \bar{u}\right] \partial_{x}+\left[-\frac{1}{R e}\left(\frac{d \bar{\mu}}{d \bar{T}}\right) \bar{T}_{y}+\bar{\rho} \bar{v}\right] \partial_{y} \\
& +\frac{4}{3 R e} \bar{\mu} \beta^{2}+\bar{\rho} \bar{u}_{x}+\bar{\rho} \bar{v}_{y}+\mathrm{i} \beta \bar{\rho} \bar{w}+\bar{u} \bar{\rho}_{x}+\bar{v} \bar{\rho}_{y} \\
\mathcal{L}_{z \hat{\theta}}= & -\frac{1}{R e}\left(\frac{d \bar{\mu}}{d \bar{T}}\right) \bar{w}_{x} \partial_{x}-\frac{1}{R e}\left(\frac{d \bar{\mu}}{d \bar{T}}\right) \bar{w}_{y} \partial_{y} \\
& -\frac{1}{R e}\left(\frac{d \bar{\mu}}{d T}\right)\left[\bar{w}_{x x}+\bar{w}_{y y}-\frac{2}{3} \mathrm{i} \beta\left(\bar{u}_{x}+\bar{v}_{y}\right)\right]-\frac{1}{R e}\left(\frac{d^{2} \bar{\mu}}{d T^{2}}\right)\left[\bar{T}_{x} \bar{w}_{x}+\bar{T}_{y} \bar{w}_{y}\right]-\frac{\bar{\rho}}{T}\left(\bar{u} \bar{w}_{x}+\bar{v} \bar{w}_{y}\right) \\
\mathcal{L}_{z \hat{p}}= & \frac{i \beta}{\gamma M^{2}}+\frac{1}{T}\left(\bar{u} \bar{w}_{x}+\bar{v} \bar{w}_{y}\right) \\
\mathcal{R}_{z \hat{w}}= & \mathrm{i} \bar{\rho}
\end{aligned}
$$


Disturbance Energy:

$$
\begin{aligned}
& \mathcal{L}_{e \hat{u}}=\left[-2 \frac{\gamma(\gamma-1) M^{2}}{R e} \bar{\mu}\left(\frac{4}{3} \bar{u}_{x}-\frac{2}{3} \bar{v}_{y}\right)+\gamma \bar{\rho} \bar{T}\right] \partial_{x}-2 \frac{\gamma(\gamma-1) M^{2}}{R e} \bar{\mu}\left(\bar{u}_{y}+\bar{v}_{x}\right) \partial_{y} \\
& -2 \frac{\text { i } \beta \gamma(\gamma-1) M^{2}}{R e} \bar{\mu} \bar{w}_{x}+\bar{T} \bar{\rho}_{x}+\bar{\rho} \bar{T}_{x} \\
& \mathcal{L}_{e \hat{v}}=-2 \frac{\gamma(\gamma-1) M^{2}}{\operatorname{Re}} \bar{\mu}\left(\bar{u}_{y}+\bar{v}_{x}\right) \partial_{x}+\left[-2 \frac{\gamma(\gamma-1) M^{2}}{\operatorname{Re}} \bar{\mu}\left(-\frac{2}{3} \bar{u}_{x}+\frac{4}{3} \bar{v}_{y}\right)+\gamma \bar{\rho} \bar{T}\right] \partial_{y} \\
& -2 \frac{\mathrm{i} \beta \gamma(\gamma-1) M^{2}}{R e} \bar{\mu} \bar{w}_{y}+\bar{T} \bar{\rho}_{y}+\bar{\rho} \bar{T}_{y} \\
& \mathcal{L}_{e \hat{w}}=-2 \frac{\gamma(\gamma-1) M^{2}}{R e} \bar{\mu} \bar{w}_{x} \partial_{x}-2 \frac{\gamma(\gamma-1) M^{2}}{R e} \bar{\mu} \bar{w}_{y} \partial_{y} \\
& +\frac{4}{3} \frac{\mathrm{i} \beta \gamma(\gamma-1) M^{2}}{R e} \bar{\mu}\left(\bar{u}_{x}+\bar{v}_{y}\right)+\mathrm{i} \beta \gamma \bar{\rho} \bar{T} \\
& \mathcal{L}_{e \hat{\theta}}=-\frac{\gamma \bar{\kappa}}{\operatorname{Re} P r} \partial_{x x}^{2}-2 \frac{\gamma}{\operatorname{RePr}}\left(\frac{d \bar{\kappa}}{d \bar{T}}\right) \bar{T}_{x} \partial_{x}-\frac{\gamma \bar{\kappa}}{\operatorname{RePr}} \partial_{y y}^{2}-2 \frac{\gamma}{\operatorname{Re} \operatorname{Pr}}\left(\frac{d \bar{\kappa}}{d \bar{T}}\right) \bar{T}_{y} \partial_{y} \\
& +\frac{\beta^{2} \gamma \bar{\kappa}}{\operatorname{Re} P r}-\frac{\gamma}{\operatorname{Re} P r}\left(\frac{d \bar{\kappa}}{d \bar{T}}\right)\left(\bar{T}_{x x}+\bar{T}_{y y}\right)-\frac{\gamma}{\operatorname{RePr}}\left(\frac{d^{2} \bar{\kappa}}{d \bar{T}^{2}}\right)\left(\bar{T}_{x}^{2}+\bar{T}_{y}^{2}\right) \\
& -\frac{\gamma(\gamma-1) M^{2}}{R e}\left(\frac{d \bar{\mu}}{d T}\right)\left(\frac{4}{3} \bar{u}_{x}^{2}+\bar{v}_{x}^{2}+\bar{w}_{x}^{2}+\bar{u}_{y}^{2}+\frac{4}{3} \bar{v}_{y}^{2}+\bar{w}_{y}^{2}-\frac{4}{3} \bar{u}_{x} \bar{v}_{y}+2 \bar{u}_{y} \bar{v}_{x}\right) \\
& \mathcal{L}_{e \hat{p}}=\bar{u} \partial_{x}+\bar{v} \partial_{y}+\mathrm{i} \beta \bar{w}+\gamma\left(\bar{u}_{x}+\bar{v}_{y}\right) \\
& \mathcal{R}_{e \hat{p}}=\mathrm{i}
\end{aligned}
$$

Disturbance Continuity:

$$
\begin{aligned}
& \mathcal{L}_{c \hat{u}}=\bar{\rho} \bar{T} \partial_{x}+\bar{T} \bar{\rho}_{x} \\
& \mathcal{L}_{c \hat{v}}=\bar{\rho} \bar{T} \partial_{y}+\bar{T} \bar{\rho}_{y} \\
& \mathcal{L}_{c \hat{w}}=\mathrm{i} \beta \bar{\rho} \bar{T} \hat{w} \\
& \mathcal{L}_{c \hat{\theta}}=-\bar{\rho} \bar{u} \partial_{x}-\bar{\rho} \bar{v} \partial_{y}-\bar{\rho} \bar{u}_{x}-\bar{\rho} \bar{v}_{y}-\mathrm{i} \beta \bar{\rho} \bar{w}-\bar{u} \bar{\rho}_{x}-\bar{v} \bar{\rho}_{y}+\frac{\bar{\rho}}{T}\left(\bar{u} \bar{T}_{x}+\bar{v} \bar{T}_{y}\right) \\
& \mathcal{L}_{c \hat{p}}^{G}=\bar{u} \partial_{x}+\bar{v} \partial_{y}+\mathrm{i} \beta \bar{w}+\bar{u}_{x}+\bar{v}_{y}-\frac{1}{\bar{T}}\left(\bar{u} \bar{T}_{x}+\bar{v} \bar{T}_{y}\right) \\
& \mathcal{R}_{c \hat{\theta}}=-\mathrm{i} \bar{\rho} \\
& \mathcal{R}_{c \hat{p}}=\mathrm{i}
\end{aligned}
$$




\section{References}

${ }^{1} \mathrm{~J}$. Alvarez, E. Kerschen, and A. Tumin. A theoretical model for cavity acoustic resonances in subsonic flow. AIAA Paper 2004-2845, 2004.

${ }^{2}$ W. Blumen. J. Fluid Mech., 40:769, 1970.

${ }^{3}$ J.P. Boyd. Chebyshev and Fourier spectral methods. Springer, 1989.

${ }^{4}$ Barkley D., Gomes M.G.M., and Henderson R.D. Threedimensional instability in flow over a backward-facing step. $J$. Fluid Mech., 473:167-190, 2002.

${ }^{5}$ N. Forestier, L. Jacquin, and P. Geffroy. The mixing layer over a deep cavity at high- subsonic speed. J. Fluid Mech., 475:101-145, 2003.

${ }^{6} \mathrm{M}$. Gharib and A. Roshko. The effect of flow oscillations on cavity drag. J. Fluid Mech., 177:501-530, 1987.

${ }^{7}$ X. Gloerfelt, C. Bailly, and D. Juve. Direct computation of the noise radiated by a subsonic cavity flow and application of integral methods. 266:119-146, 2003.

${ }^{8}$ X. Gloerfelt, C. Bogey, C. Bailly, and D. Juve. Aerodynamic noise induced by laminar and turbulent boundary layers over rectangular cavities. AIAA Paper 2002-2476, 2002.

${ }^{9}$ D. Kosloff and H. Tal-Ezer. Modified Chebyshev pseudospectral methods with $o\left(N^{-1}\right)$ time-step restriction. J. Comput. Phys., 104:457-469, 1993.

${ }^{10}$ M.G. Macaraeg, C.L. Streett, and M.Y. Hussaini. Technical report.

${ }^{11}$ L. M. Mack. Boundary layer linear stability theory. In AGARD-R-709 Special course on stability and transition of laminar flow, pages 3.1-3.81, 1984.

${ }^{12}$ L. Rosenhead. Laminar Boundary Layers. Oxford University Press, 1963.

${ }^{13}$ C. Rowley, T. Colonius, and A. Basu. On self-sustained oscillations in two-dimensional compressible flow over rectangular cavities. J. Fluid Mech., 455:315-346, 2002.

${ }^{14}$ C. M. Shieh and P. J. Morris. Comparison of two- and three-dimensional turbulent cavity flows. AIAA Paper 20010511, 2001.

${ }^{15} \mathrm{~T}$. Tatsumi and T. Yoshimura. Stability of the laminar flow in a rectangular duct. J. Fluid Mech., 212:437-449, 1990.

${ }^{16} \mathrm{~V}$. Theofilis. Linear instability in two spatial dimensions. In K. D. Papailiou, editor, Fourth European Computational Fluid Dynamics Conference ECCOMAS'98, pages 547-552, Chichester, N. York, 1998. J. Wiley and Sons.

${ }^{17} \mathrm{~V}$. Theofilis. Globally-unstable flows in open cavities. page 12 pp. AIAA Paper 2000-1965, 2000.

${ }^{18} \mathrm{~V}$. Theofilis. Global instabilities and control of nonparallel flows. AIAA Paper 2002-3279, 2002.

${ }^{19} \mathrm{~V}$. Theofilis. Advances in global linear instability of nonparallel and three-dimensional flows. Prog. Aero. Sciences, 39 (4):249-315, 2003.

${ }^{20} \mathrm{~V}$. Theofilis and T. Colonius. An algorithm for the recovery of 2- and 3-d biglobal instabilities of compressible flow over 2-d open cavities. AIAA Paper 2003-4143, 2003.

${ }^{21}$ V. Theofilis, P. W. Duck, and J. Owen. Viscous linear stability analysis of rectangular duct and cavity flows. J. Fluid Mech., 505:249-286, 2004.

${ }^{22}$ V. Theofilis, A. Fedorov, D. Obrist, and U. Ch. Dallmann. The extended Görtler-Hämmerlin model for linear instability of three-dimensional incompressible swept attachment-line boundary layer flow. J. Fluid Mech., 487:271-313, 2003. 